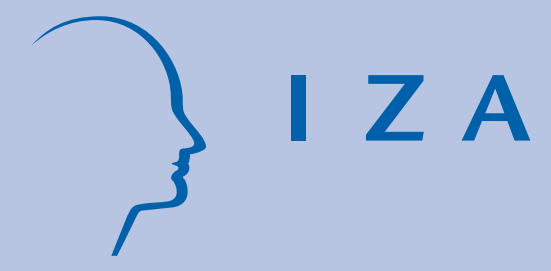

IZA Standpunkte Nr. 78

Der Wandel der Erwerbsformen in Deutschland

Werner Eichhorst

Februar 2015

Forschungsinstitut zur Zukunft der Arbeit Institute for the Study of Labor 


\title{
Der Wandel der Erwerbsformen in Deutschland
}

\author{
Werner Eichhorst \\ $I Z A$
}

\section{IZA Standpunkte Nr. 78 Februar 2015}

IZA

Postfach 7240

53072 Bonn

Tel.: (0228) 3894-0

Fax: (0228) 3894-180

E-Mail: iza@iza.org

Die Schriftenreihe „IZA Standpunkte” veröffentlicht politikrelevante Forschungsarbeiten und Diskussionsbeiträge von IZA-Wissenschaftlern, IZA Research Fellows und IZA Research Affiliates in deutscher Sprache. Die Autoren sind für den Inhalt der publizierten Arbeiten verantwortlich. Im Interesse einer einheitlichen Textzirkulation werden Aktualisierungen einmal publizierter Arbeiten nicht an dieser Stelle vorgenommen, sondern sind gegebenenfalls nur über die Autoren selbst erhältlich. 
IZA Standpunkte Nr. 78

Februar 2015

\section{ZUSAMMENFASSUNG}

\section{Der Wandel der Erwerbsformen in Deutschland}

Der vorliegende Beitrag untersucht den Wandel der Erwerbsformen in Deutschland und zeigt, dass atypische Beschäftigung in bestimmten Sektoren und Berufen zum Aufbau von Erwerbstätigkeit beigetragen hat, gleichzeitig ist jedoch das so genannte Normalarbeitsverhältnis nach wie vor die bei weitem am deutschen Arbeitsmarkt dominante Erwerbsform aber auch dort haben verschiedene Formen der Flexibilisierung Raum gegriffen. Insgesamt ist die Vielfalt der Erwerbs- und Arbeitsformen in Deutschland gewachsen.

JEL-Codes: J21, J31, J58

Schlagworte: atypische Beschäftigung, Normalarbeitsverhältnis, Deutschland, Flexibilisierung

Kontaktadresse:

Werner Eichhorst

IZA

Postfach 7240

D-53072 Bonn

E-mail: Eichhorst@iza.org 
1. Flexiblere Arbeitswelten - Externe und Interne Flexibilität

Der Arbeitsmarkt und die Arbeitswelt in Deutschland befinden sich in einem schon länger andauernden Veränderungsprozess, der in der öffentlichen und fachlichen Diskussion häufig mit dem Begriff einer zunehmenden „Flexibilisierung“ oder „Entgrenzung“ der Arbeit beschrieben wird. Die „Entgrenzung“ der Arbeit lässt sich als eine Auflösung traditioneller räumlicher, zeitlicher oder organisatorischer Grenzen von Arbeit verstehen, insbesondere als Aufweichung und Verschiebung der Grenzen zwischen Arbeit und Privatleben, sodass sich beide Bereiche stärker durchdringen (Voß 1998, Gottschall und Voß 2005). Die Arbeitsmarktforschung umschreibt dies auch mit dem Begriff „Flexibilisierung“. Flexibilität kann sich sowohl auf innerbetriebliche Abläufe als auch auf die Struktur der Unternehmen und ihrer Belegschaften beziehen - dies bedeutet auch hier eine Öffnung tradierter Grenzziehungen. Antriebskräfte für diese Entwicklung sind die Globalisierung, technologische Innovationen, die Flexibilisierung der institutionellen Regeln auf den Arbeitsmärkten sowie der strukturelle Wandel hin zum Dienstleistungssektor, aber auch veränderte Lebenslagen und Präferenzen der Erwerbstätigen. Damit gehen neue Formen flexiblen Arbeitens in einem insgesamt dynamischeren und verschärften Wettbewerb einher. Es ist dabei sinnvoll zwischen innerbetrieblichen Formen der Flexibilisierung und Entgrenzung auf der einen Seite und einer Neudefinition der Grenzen von Unternehmen auf der anderen Seite durch veränderte Beziehungen zwischen dem betrieblichen Kern und einem flexibleren Randbereich zu unterscheiden, wenngleich sich beide Entwicklungen überlagern und gegenseitig beeinflussen. 


\subsection{Externe Flexibilität}

In den letzten zwei Jahrzehnten hat die „externe“ Flexibilität, sprich die Verbreitung atypischer Beschäftigung, in Deutschland zugenommen, ist jedoch in den letzten Jahren zum Stillstand gekommen. Hierzu zählen befristete Arbeitsverträge sowie verschiedene Formen der Teilzeitarbeit (sozialversicherte Teilzeit und geringfügige Beschäftigung (Minijobs)), aber auch Zeitarbeit und Selbstständigkeit mit abhängig Beschäftigten und ohne abhängig Beschäftigte (Freelancer). Ein Blick auf die Entwicklung der erwerbsfähigen Bevölkerung nach Erwerbsstatus macht dies deutlich (Abbildung 1). Der Anteil der inaktiven Bevölkerung nimmt seit Mitte der 2000er Jahre ab. Immer mehr Erwerbsfähige finden eine Beschäftigung, und dabei spielen flexible Beschäftigungsformen eine entscheidende Rolle. Diese Entwicklung ist nicht nur in Deutschland, sondern auch in vielen anderen europäischen Staaten zu beobachten. Vor allem der Anteil an sozialversicherter Teilzeit hat zugenommen. Darunter ist ein Normalarbeitsverhältnis mit reduzierter Stundenzahl zu verstehen. Ebenfalls ist die Beschäftigung in Minijobs (Eichhorst et al. 2012) gewachsen sowie, auf einem relativ begrenzten Niveau, die Zeitarbeit. 


\section{Abbildung 1: Erwerbsfähige Bevölkerung in Deutschland nach Erwerbsstatus}

(1992-2012)

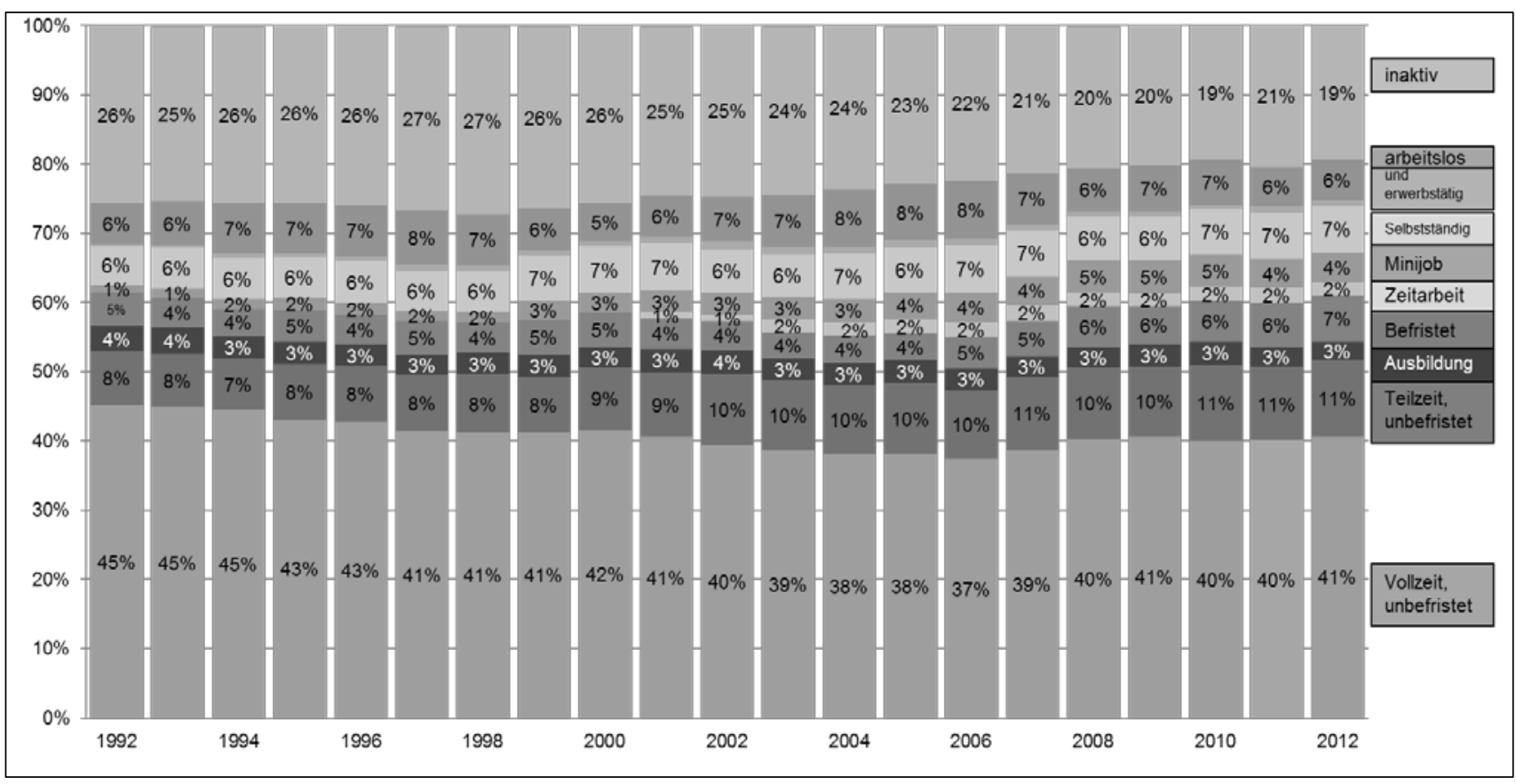

Quelle: SOEP 1992-2012, eigene Berechnungen.

Zwar hat der Anteil an unbefristeten Vollzeitarbeitsverträgen seit 1992 leicht abgenommen, jedoch ist der Bestand insgesamt seit 2000 relativ stabil geblieben. So ist auch in 2012 das unbefristete Vollzeitarbeitsverhältnis (Normalarbeitsverhältnis) mit 41 Prozent aller Personen im erwerbsfähigen Alter oder etwa 60 Prozent aller Beschäftigten immer noch die häufigste Erwerbsform (Eichhorst und Tobsch 2013 und 2014).

Die Entwicklung hin zu mehr atypischer Beschäftigung liegt zu einem im Strukturwandel hin zum oft eher kleinbetrieblich organisierten privaten Dienstleistungssektor, der stärker auf bestimmte externe Flexibilitätsformen angewiesen ist, und in der Verstärkung des Wettbewerbsdrucks in einer zunehmend globalisierten Volkswirtschaft. Aber auch die Politik hat durch den Abbau rechtlicher Schranken für bestimmte flexible Beschäftigung in den letzten Jahren die Nutzung erleichtert. Dies ermöglicht es den Unternehmen, Arbeitsplätze in Form flexibler Arbeitsverhältnisse zu schaffen (Eichhorst und Marx 2011). Nicht zu vergessen ist auch die zunehmende Nachfrage nach Teilzeitjobs auf Grund der zunehmenden Erwerbstätigkeit der Frauen. 
Abbildung 2 gibt einen Einblick in die Beschäftigungsdynamik der gesamten Arbeitsmarkveränderung in Deutschland in beruflicher Perspektive. Sie zeigt die Entwicklung des Anteils atypischer Jobs an allen Arbeitsplätzen, differenziert nach Berufsgruppen der Klassifikation ISCO-88, auf der einen Seite (y-Achse) und die Entwicklung der Beschäftigung in diesen Berufen von 1995 bis 2011 auf der anderen Seite (x-Achse). Die Entwicklung ist geprägt von einem gewissen Auseinanderdriften.

\section{Abbildung 2: Entwicklung von Berufsgruppen und atypischer Beschäftigung (1995-2011)}

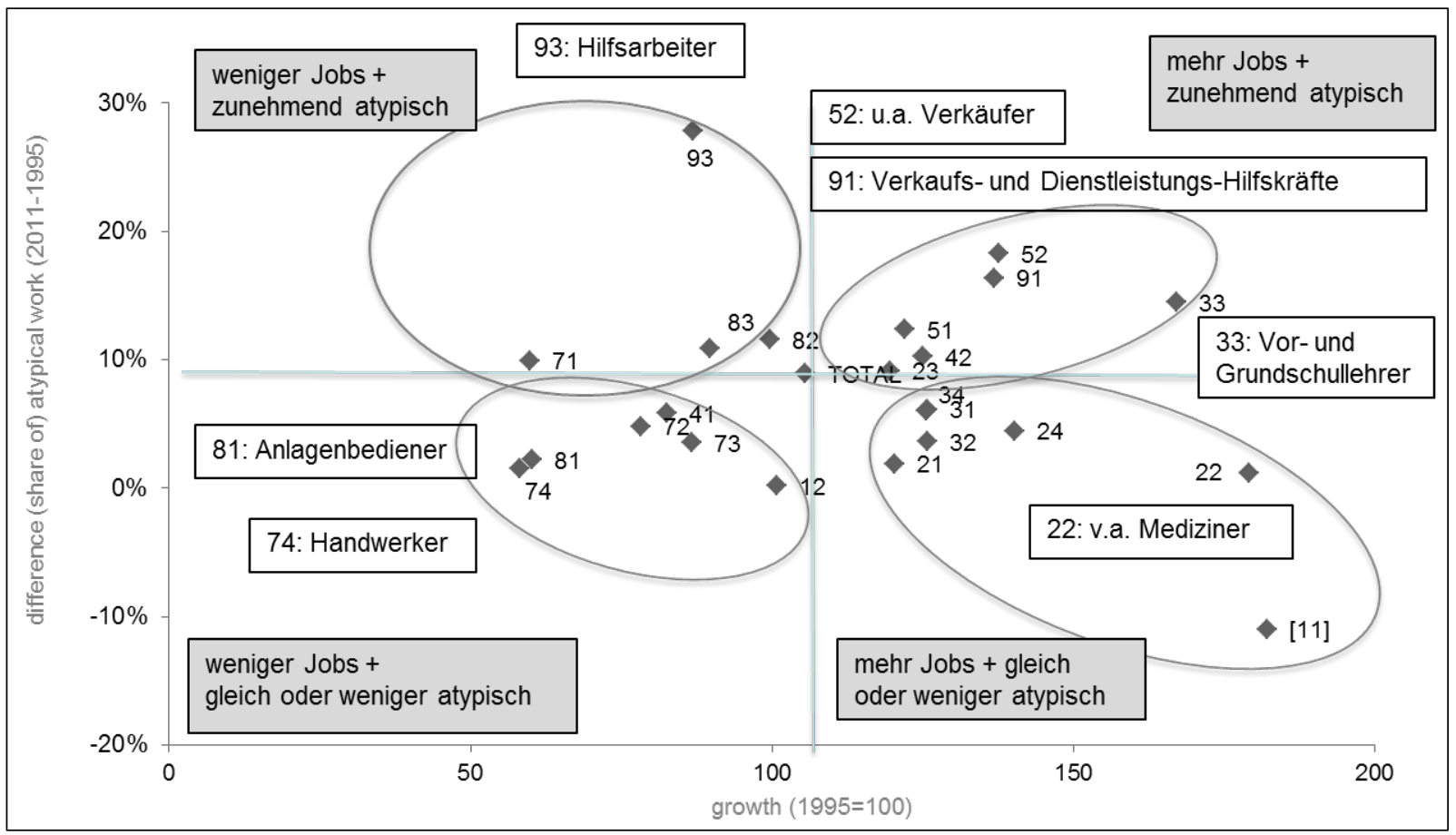

Quelle: SOEP, eigene Berechnungen.

Vor allem die Beschäftigung in hoch qualifizierten Berufen (z.B. Naturwissenschaftler, Mediziner, Wissenschaftler und Leitungskräfte ganz generell) wächst, während der Anteil atypischer Beschäftigung konstant bleibt oder sogar abnimmt (Gruppen 22, 24, 31, 32, 34 nach ISCO-88). Auch die Arbeitsbedingungen von Büroangestellten (41) und Fertigungsberufen $(71,72,73)$ haben sich kaum verändert, während die Beschäftigung in diesen Berufen abnahm. Dagegen stiegen sowohl die Beschäftigung insgesamt wie auch der Anteil an atypischer Beschäftigung vor allem bei mittleren und niedrig qualifizierten Personal sowie in Dienstleistungsberufen (42, 51, 52, 91) erheblich an. Eine ähnliche Entwicklung, jedoch etwas weniger ausgeprägt, ist bei nichtwissenschaftlichen Lehrkräften (33) zu beobachten. In 
einigen niedrig qualifizierten Fertigungsberufen wie zum Beispiel bei Arbeitern (93) ist die Beschäftigung zurückgegegangen, während der Anteil an atypischer Beschäftigung gewachsen ist.

Tabelle 1: Atypische Beschäftigung in ausgewählten Berufen

\begin{tabular}{|c|c|c|c|c|c|c|c|c|}
\hline & $\begin{array}{l}\text { Wissen } \\
\text { schaftler }\end{array}$ & $\begin{array}{l}\text { Kreativ } \\
\text { berufe }\end{array}$ & Pflege & $\begin{array}{c}\text { Fach } \\
\text { arbeiter }\end{array}$ & $\begin{array}{c}\text { Reinigungs } \\
\text { kräfte }\end{array}$ & $\begin{array}{c}\text { Hilfs } \\
\text { arbeiter }\end{array}$ & $\begin{array}{c}\text { Bank- und } \\
\text { Versicherungs } \\
\text { berufe }\end{array}$ & gesamt \\
\hline Anteil an der Gesamtbeschäftigung & $0,4 \%$ & $1,5 \%$ & $4,1 \%$ & $6,1 \%$ & $3,8 \%$ & $2,0 \%$ & $2,0 \%$ & $100,0 \%$ \\
\hline Befristete Verträge & $38 \%$ & $25 \%$ & $23 \%$ & $18 \%$ & $12 \%$ & $14 \%$ & $9 \%$ & $15 \%$ \\
\hline \multicolumn{9}{|l|}{ Davon } \\
\hline Berufsausbildung & $14 \%$ & $31 \%$ & $35 \%$ & $74 \%$ & $4 \%$ & $\ldots$ & $70 \%$ & $40 \%$ \\
\hline unfreiwillig & $19 \%$ & $14 \%$ & $15 \%$ & $8 \%$ & $35 \%$ & $35 \%$ & $\ldots$ & $16 \%$ \\
\hline Teilzeit & $19 \%$ & $17 \%$ & $33 \%$ & $2 \%$ & $30 \%$ & $12 \%$ & $20 \%$ & $17 \%$ \\
\hline Minijob & $9 \%$ & $9 \%$ & $14 \%$ & $1 \%$ & $41 \%$ & $15 \%$ & $2 \%$ & $9 \%$ \\
\hline Zeitarbeiter & $0 \%$ & $0 \%$ & $\cdots$ & $3 \%$ & $\cdots$ & $9 \%$ & $0 \%$ & $2 \%$ \\
\hline Selbstständige & $\ldots$ & $4 \%$ & $0 \%$ & $3 \%$ & $1 \%$ & $\ldots$ & $\ldots$ & $5 \%$ \\
\hline Freelancer & $13 \%$ & $42 \%$ & $3 \%$ & $2 \%$ & $3 \%$ & $\ldots$ & $1 \%$ & $6 \%$ \\
\hline$<67 \%$ des Medianlohns & $\ldots$ & $\ldots$ & $37 \%$ & $22 \%$ & $52 \%$ & $39 \%$ & $15 \%$ & $22 \%$ \\
\hline Anteil Frauen & $35 \%$ & $51 \%$ & $86 \%$ & $4 \%$ & $72 \%$ & $45 \%$ & $62 \%$ & $48 \%$ \\
\hline Betrieb < 11 & $\ldots$ & $16 \%$ & $36 \%$ & $11 \%$ & $29 \%$ & $6 \%$ & $17 \%$ & $19 \%$ \\
\hline Betrie b > 49 & $84 \%$ & $55 \%$ & $33 \%$ & $65 \%$ & $40 \%$ & $71 \%$ & $61 \%$ & $55 \%$ \\
\hline Gering qualifziert (ISCED 0-2) & $\ldots$ & $5 \%$ & $14 \%$ & $18 \%$ & $39 \%$ & $43 \%$ & $4 \%$ & $15 \%$ \\
\hline Hoch qualifiziert (ISCED 5-6) & $88 \%$ & $50 \%$ & $13 \%$ & $9 \%$ & $5 \%$ & $4 \%$ & $21 \%$ & $25 \%$ \\
\hline
\end{tabular}

Quelle: Mikrozensus 2009, Scientific Use File, eigene Berechnungen (Eichhorst, Marx und Tobsch, 2013).

In Tabelle 1 ist der Anteil atypischer Beschäftigung nach ausgewählten Berufsgruppen der Klassifikation ISCO-88 (Wissenschaftler 231, Kreativberufe 245 und 347, Pflege 513 und 346, Facharbeiter 721-724, Reinigungskräfte 913, 914 und 916, Hilfsarbeiter 932, Bank- und Versicherungsberufe 412) dargestellt. Befristete Verträge sind vor allem bei Wissenschaftlern mit einem Anteil von 38 Prozent (der Gesamtbeschäftigung), sowie in Kreativberufen und Pflegeberufen mit einem Anteil von mehr als 20 Prozent verbreitet. Auch bei Facharbeitern liegt der Anteil noch bei 18 Prozent, jedoch werden hier befristete Verträge meist zu Ausbildungszwecken verwendet (ähnlich wie in Bank- und Versicherungsberufen). Der Anteil bei Reinigungskräften (12 Prozent) und Hilfsarbeitern (14 Prozent) liegt etwas niedriger, jedoch arbeiten in diesen Berufen mehr als ein Drittel unfreiwillig in atypischer Beschäftigung. Teilzeit findet man häufig im Pflegebereich und bei Reinigungskräften mit ungefähr einem Drittel, kaum dagegen bei Facharbeitern. Über 40 Prozent der Reinigungskräfte, sprich jede zweite von fünf, ist in einem Minijob beschäftigt, 
welcher mit niedrigen Monatslöhnen verbunden ist sowie keiner Sozialversicherung beinhaltet. Auch Hilfsarbeiter und Pflegekräfte sind häufig geringfügig Beschäftigte. Die Verbreitung von Zeitarbeit ist in Deutschland mit nur 2 Prozent kaum verbreitet. Am häufigsten ist sie in der Industrie zu finden. Der Anteil an Zeitarbeit ist bei Hilfsarbeitern mit 9 Prozent am größten (bei Facharbeitern nur 3 Prozent). Freelancer (Selbstständige ohne weitere Arbeitskräfte) sind vor allem in Kreativberufen zu finden. Weiterhin sind unter Wissenschaftlern einige Freelancer zu finden, in anderen Berufsgruppen jedoch kaum.

Niedrigverdiener ist, wer weniger als 67 Prozent vom Medianlohn verdient. Fast ein Viertel der deutschen Arbeitnehmer sind Niedrigverdiener. Hierbei sind vor allem die niedrig-qualifizierten Berufe wie Reinigungskräfte und Hilfsarbeiter betroffen. Obwohl Pflegekräfte in Deutschland eine berufliche Ausbildung durchlaufen, liegt deren Niedrigverdiener-Anteil bei 37 Prozent.

2013 haben Eichhorst, Marx und Tobsch in einer multivariaten Regression (SOEP Daten 2005-2011) für Deutschland gezeigt, dass die Wahrscheinlichkeit, in einem atypischen Arbeitsverhältnis beschäftigt zu sein, für jüngere und ältere Arbeitskräfte, für Frauen (vor allem mit Kindern), für niedrig-qualifizierte Personen sowie in niedrigqualifizierten Berufen besonders hoch ist. Weiterhin ist es auch in kleinen Firmen wahrscheinlicher atypisch beschäftigt zu sein als in großen Firmen. Jedoch ist die Wahrscheinlichkeit, in einem befristeten Vertrag angestellt zu sein, größer in mittelständischen und großen Unternehmen, da der individuelle Kündigungsschutz abhängig von der Firmengröße ist. In Teilzeit finden sich vor allem verheiratete Frauen und insbesondere Mütter. Auch mittel bis hoch-qualifizierte Personen sind häufiger in befristeten Verträgen angestellt, jedoch vor allem zu Ausbildungszwecken oder auf Grund des höheren Anteils befristeter Verträge bei akademischen Jobs im öffentlichen Bereich.

Zusammenfassend kann man sagen, dass atypische Beschäftigung besonders im hoch qualifizierten kreativen Bereich (Freelancers), im Gesundheits- und Sozialwesen sowie im akademisch-wissenschaftlichen Bereich (Teilzeit, Befristungen), bei einfacheren Tätigkeiten im Dienstleistungssektor (Minijobs) sowie in der verarbeitenden Industrie (Zeitarbeit) bis vor kurzem zugenommen hat. Jedoch ist in vielen Bereichen des mittleren und höheren Qualifikationssegments immer noch der unbefristete Vollzeitvertrag die vorherrschende Anstellungsform. Die Bedeutung 
atypischer oder niedrig entlohnter Erwerbsformen folgt dabei im Grunde dem Angebot und der Nachfrage nach Arbeitskräften mit den jeweiligen Qualifikationen, wird aber auch von der Rolle sektoraler Tarifpartner und gesetzlichen Regelungen beeinflusst (Eichhorst, Marx und Tobsch 2013). Meistens ist die Bezahlung von Arbeitnehmern in atypischen Beschäftigungsverhältnissen geringer als bei vergleichbarer unbefristeter Vollzeitarbeit (Tabelle 2).

\section{Tabelle 2: Atypische Beschäftigung, Qualifikation und Niedriglohn}

\begin{tabular}{|c|c|c|c|c|c|c|c|}
\hline \multirow{2}{*}{$\begin{array}{l}\text { Anteil der Niedrigbezieher in \% (über } \\
50 \% \text { in Schriftschnitt fett) }\end{array}$} & \multirow[b]{2}{*}{ Gesamt } & \multirow{2}{*}{$\begin{array}{l}\text { Normalarbeits- } \\
\text { verträge (VZ } \\
\text { und TV > 20h) }\end{array}$} & \multicolumn{5}{|c|}{ Atypische Erwerbsformen } \\
\hline & & & Gesamt & Teilzeit & Befristung & Minijobs & Zeitarbeit \\
\hline Gesamt & 20,6 & 10,8 & 49,8 & 20,9 & 33,5 & 84,3 & 67,7 \\
\hline Frauen & 26,5 & 15,1 & 47,6 & 19,2 & 35,5 & 84,8 & 72,9 \\
\hline Männer & 15,8 & 8,1 & 53,7 & 34,3 & 31,6 & 83,4 & 65,4 \\
\hline Geringe Qualifikation & 52,8 & 22,7 & 77,8 & 44,7 & 62,2 & 88,1 & 85,5 \\
\hline Mittlere Qualifikation & 17,7 & 12,1 & 39,4 & 17,1 & 36,2 & 77,2 & 57,6 \\
\hline Hohe Qualifikation & 1,7 & 0,5 & 8,3 & 2,7 & 5,7 & 61,4 & 20,7 \\
\hline \multicolumn{8}{|l|}{ Ausgewählte Berufsgruppen } \\
\hline Akademische Berufe & 3,0 & 1,0 & 10,5 & 3,5 & 5,7 & 64,9 & I \\
\hline Techniker und gleichwertige Berufe & 7,6 & 4,0 & 24,9 & 4,7 & 17,3 & 68,1 & 29,2 \\
\hline Büroangestellte & 23,4 & 10,9 & 48,1 & 13,2 & 39,4 & 80,3 & 62,5 \\
\hline Verkaufs- und Dienstleistungspersonal & 42,3 & 28,4 & 65,4 & 30 & 58,3 & 88,6 & 72,1 \\
\hline Handwerk & 16,1 & 11,1 & 48,7 & 32,6 & 31,3 & 81,0 & 47,6 \\
\hline Maschinenbediener und Monteure & 23,7 & 17,1 & 60,6 & 33,2 & 39,7 & 89,3 & 63,0 \\
\hline Hilfsarbeiter & 61,5 & 39,7 & 79,2 & 56,3 & 70,7 & 90,3 & 89,2 \\
\hline
\end{tabular}

Quelle: Destatis.

Besonders betroffen sind Arbeitnehmer im privaten Dienstleistungssektor mit mittlerer oder geringer Qualifikation, vor allem wenn es sich hierbei um einen Minijob oder Zeitarbeit handelt. Zum Teil kann dies durch eine geringere Betriebszugehörigkeit bzw. Berufserfahrung oder auch auf Grund unterschiedlicher Tätigkeitsprofile erklärt werden. Auch bei Vollzeitbeschäftigten sind wachsende Unterschiede bei der Entlohnung zu beobachten (OECD, 2013). Diese Entwicklung kann durch die Deregulierung atypischer Arbeitsformen erklärt werden, ebenso durch den Rückgang der Tarifbindung und die Verschiebung hin zum privaten Dienstleistungssektor, der stärker atypische Beschäftigung nutzt und seltener von Tarifverträgen abgedeckt wird (Dustmann, Ludsteck und Schönberg 2009).

Die wachsende Zergliederung der Wertschöpfungsketten in Unternehmen führt dazu, dass die Grenzen von Unternehmen neu definiert werden müssen, da in bestimmten 
Bereichen vermehrt auf externe Dienstleister über Werkverträge, Selbstständige oder Zeitarbeit zurückgegriffen wird. Wie schon erwähnt werden Zeitarbeiter vor allem in der verarbeitenden Industrie eingesetzt, aber ihr Anteil an der erwerbsfähigen Bevölkerung beträgt lediglich etwa 2 Prozent. Das Wachstum der Freelancer auf über zwei Mio. konzentriert sich auf bestimmte hoch-qualifizierte Bereiche wie IT, Medien und Beratung. Auch externe Dienstleister, die über Werkverträge eingesetzt werden, sind besonders für anspruchsvollere und spezialisierte Tätigkeiten, wie im Bereich von Forschung und Entwicklung, IT, Beratungs- und Finanzdienstleistungen, sowie in Bereichen wie Logistik, Facility-Management und Teilen der gewerblichen Produktion, von Bedeutung (Klebe 2013, Hofmann und Nøstdal 2014). Aber auch hier entstehen Normalarbeitsverhältnisse mit teilweise durchaus vergleichbar guten Arbeitsbedingungen, nur eben nicht in direkter Anstellung beim Kunden. Weiterhin gibt es auch Werkverträge im Dienstleistungssektor für einfache Tätigkeiten.

Insgesamt führt diese Entwicklung zu einem erheblichen Wachstum von unternehmensbezogenen Dienstleistungen. Viele Unternehmen lagern Tätigkeiten aus und lassen diese von spezialisierten Zulieferbetrieben oder freien Mitarbeitern erstellen, wenn die notwendigen Humanressourcen auf dem externen Markt günstiger, flexibler einsetzbar oder leichter verfügbar sind als im eigenen Unternehmen („buy“ statt „make“). In diesem Zusammenhang kann man von einem Wachstum der „Projektwirtschaft“ (Hofmann, Rollwagen und Schneider 2007; Rump et al. 2010) sprechen. Das führt zu höheren Vorleistungs- und Importanteilen sowohl bei Gütern als auch bei Dienstleistungen sowie tendenziell auch zu einem höheren Anteil bei der Wertschöpfung im Ausland. Fest abgegrenzte Unternehmen lösen sich also wenigstens in bestimmten Bereichen tendenziell auf und schaffen größere Netzwerke innerhalb und außerhalb zum Austausch von Teilerzeugnissen oder Dienstleistungen. Das resultiert in komplexeren Zulieferbeziehungen, Werkvertragsketten, Subunternehmen (national und grenzüberschreitend), Wertschöpfungsketten und -netzwerken.

Durch die stärkere Verbreitung atypischer Beschäftigungsverhältnisse und Niedriglohnjobs ist der deutsche Arbeitsmarkt insgesamt flexibler und damit aufnahmefähiger geworden. Aber gleichzeitig findet man eine bemerkenswerte Stabilität bei der Betriebszugehörigkeit in Deutschland (Tabelle 3). Die Beschäftigungsdauer ist in den Betrieben nicht zurückgegangen. So bleibt die 
Betriebszugehörigkeit in Deutschland sehr stabil. Seit 2000 sind kontinuierlich etwa 60 Prozent der Arbeitnehmer über fünf Jahre im Unternehmen beschäftigt und rund 15 Prozent weniger als ein Jahr. Insgesamt ist die durchschnittliche Verweildauer im Betrieb sogar gestiegen. Dies lässt sich erklären durch die Abschaffung von verschiedenen Formen der Frühverrentung in der Vergangenheit. Ältere Arbeitskräfte mit längerer Betriebszugehörigkeit verweilen länger im Unternehmen.

Tabelle 3: Betriebszugehörigkeit von Beschäftigten in Deutschland

\begin{tabular}{|c|c|c|c|c|c|c|c|}
\hline & $\begin{array}{c}\text { Unter einem } \\
\text { Monat }\end{array}$ & $\mathbf{1}<\mathbf{6}$ Monate & $\mathbf{6}<\mathbf{1 2}$ Monate & $\mathbf{1}<\mathbf{3}$ Jahre & $\mathbf{3}<\mathbf{5}$ Jahre & $\mathbf{5}<\mathbf{1 0}$ Jahre & $>\mathbf{1 0}$ Jahre \\
\hline $\mathbf{2 0 0 0}$ & 3,4 & 4,7 & 7,5 & 14 & 11,4 & 20,2 & 38,9 \\
\hline $\mathbf{2 0 0 5}$ & 2,7 & 4,4 & 6,7 & 12,2 & 13,1 & 19,8 & 41,2 \\
\hline $\mathbf{2 0 1 1}$ & 3,1 & 5,1 & 7,5 & 12,6 & 12,2 & 16,3 & 43,2 \\
\hline
\end{tabular}

Quelle: OECD.

Durch die Steigerung der externen Flexibilität konnten mehr Menschen in Beschäftigung gebracht werden, was man an der sinkenden Zahl der inaktiven Erwerbsbevölkerung sieht. Atypische Beschäftigung erleichtert den Zugang zum Arbeitsmarkt. Ohne diesen flexiblen Rand gäbe es vermutlich weniger Zugänge in Arbeit. Jedoch erhöhen atypische Arbeitsverhältnisse auch das Risiko in instabiler und gering entlohnter Beschäftigung $\mathrm{zu}$ verbleiben, besonders bei Geringqualifizierten. Der Übergang in stabilere oder auch höher entlohnte Beschäftigung ist abhängig von Qualifikation des Arbeitsnehmers, der Arbeitszeit, dem Wirtschaftszweig und vielem mehr. In vielen Fällen verbleiben Arbeitnehmer in atypischer Beschäftigung oder durchlaufen Zyklen kurzfristiger Wechsel zwischen Beschäftigung und Arbeitslosigkeit. Generell findet man geringe Aufstiegschancen und -anreize in Bereichen, in denen die Arbeitgeber (und teilweise auch Arbeitnehmer) Anreize zum Verbleib in „atypischen“ Arbeitsverhältnissen haben. Dies gilt vor allem für Minijobs als Hinzuverdienst im Haushalt, für erwerbstätige Bezieher von Grundsicherung, sog. „Aufstocker" in Teilzeit oder Minijob, für viele Zeitarbeiter sowie für die befristet Beschäftigten im öffentlichen Bereich. 


\subsection{Interne Flexibilität}

Auch im Bereich der Normalarbeitsverhältnisse zeichnet sich eine zunehmende Flexibilisierung und Entgrenzung ab, die interne Flexibilität steigt. Das bedeutet, dass der Arbeitseinsatz innerhalb von Unternehmen vermehrt auch bei kontinuierlicher Betriebszugehörigkeit flexibler gestaltet wird bei. Interne Flexibilität kann jedoch auch bei atypischen Beschäftigungsformen auftreten. Diese steigende Flexibilität kann durch die zunehmende Verbreitung flexibler Arbeitszeiten und ungewöhnlicher Arbeitszeitmuster, durch ein stärkeres Durchdringen von Arbeitszeit und Freizeit sowie durch die verstärkte Möglichkeit des mobilen Arbeitens erreicht werden. Hierzu zählen auch flexible Projektstrukturen innerhalb der Betriebe und quasiunternehmerische Entgeltsysteme für abhängig Beschäftigte. Innerbetriebliche Projektwirtschaft ist vor allem in den Bereichen IT, Marketing, Vertrieb sowie Forschung und Entwicklung verbreitet (Rump et al. 2010).

Überlange Arbeitszeiten haben nicht unbedingt zugenommen, nur etwa acht Prozent der Arbeitnehmer leistet Überstunden und etwa fünf Prozent arbeiten mehr als 48 Stunden pro Woche. In 2011 lag der Überstundendurchschnitt bei 8,6 Stunden. Dagegen haben ungewöhnliche Arbeitszeitmuster, wie am Wochenende, nachts und in Schichten arbeiten, zugenommen (Bundesregierung 2013). In 2011 arbeitete jeder Vierte ständig oder regelmäßig an einem der beiden Wochenendtagen, was einen Anstieg von ungefähr 600.000 Beschäftigten gegenüber 2010 ist. Auch die Nachtarbeit ist auf 9,4 Prozent in 2011 im Vergleich zu 7,8 Prozent in 2001 angestiegen. Weiterhin ist der Anteil der Schichtarbeit auf 17,1 Prozent in 2011 gestiegen, was einen Zuwachs von 24 Prozent seit 2001 entspricht. Das kann vor allem auf das Wachstum von Arbeitsplätzen im privaten Dienstleistungsbereich (Verkehr und Logistik, Handel und Gastronomie), sowie in der verarbeitenden Industrie und im Gesundheits- und Sozialwesen zurück geführt werden. Auf Grund von organisatorischen Veränderungen und technologischen Innovation hat neben der Arbeitszeit auch die Erreichbarkeit außerhalb der üblichen Arbeitszeit und fester Arbeitsorten zugenommen (Badura et al. 2012). Von dieser Entwicklung sind 
besonders Führungskräfte betroffen, die auch zunehmend abends, am Wochenende oder auch im Urlaub erreichbar sind (DFK 2013).

Eine Studie des DGB (2012) besagt, dass eine Mehrheit von Beschäftigten über wachsenden Zeitdruck und Erreichbarkeit außerhalb der Arbeitszeiten berichtet. Hierbei sind Vollzeitbeschäftigte, Führungskräfte und Beschäftigte im Dienstleistungsbereich stark betroffen. Der letzte Stressreport von Lohmann-Haislah (2012) konnte dagegen keine deutliche Veränderung, sowohl für flexible und atypische Arbeitszeiten als auch für belastende Phänomene der Arbeitswelt wie Multitasking, häufige Unterbrechungen oder Zeitdruck, seit Mitte der 2000er Jahre feststellen. Die Arbeitsintensität hat sich eher auf einem hohen Niveau stabilisiert und ist bei Vollzeitbeschäftigten und Führungskräften tendenziell höher. Zwar nehmen die Klagen über Stress zu, jedoch ist Überforderung kein generelles Problem (eher quantitativ als qualitativ). Auf der anderen Seite ermöglichen flexible Arbeitszeiten und -orte, eine bessere Vereinbarung von Privat-bzw. Familienleben mit der Arbeit. Eine Studie von Hammermann und Stettes (2013) des IW Köln zeigt mit Daten des European Working Condition Survey 2010, dass Faktoren wie Arbeitszeit, Zeitdruck und Stress in Deutschland nicht signifikant für die Bewertung der Arbeitsqualität durch Arbeitnehmer sind. So geben 85 Prozent der befragten Beschäftigten an (sehr) zufrieden mit ihrer Arbeit zu sein, obwohl sie häufig hoher Geschwindigkeit und Stress am Arbeitsplatz begegnen.

Zusammenfassend kann man sagen, dass die Arbeitszeiten auf einem hohen Niveau flexibel sind, es jedoch keinen klaren Trend zu massiver „Entgrenzung“ gibt. Wie auch bei der externen Flexibilität gibt es hierbei Unterschiede je nach Wirtschafszweig sowie Berufsgruppen.

\subsection{Zwischenfazit}

Nach wie vor liegt der Kernbereich des Arbeitsmarktes beim Normalarbeitsverhältnis, soweit berufs- und betriebsspezifische Erfahrungen und Kenntnisse für die Unternehmen wichtig sind und nicht extern zu beschaffen sind. Aber auch diese unbefristeten Vollzeitarbeitsverträge sind in ihrer Gestaltung (Arbeitszeit, Arbeitsorganisation und Entlohnung) flexibler geworden und so nimmt die 
Arbeitsintensität und „Entgrenztheit“ auch in diesen Berufen zu. Gerade der Kern der Industrie ist jedoch bemerkenswert stabil, was Anzahl und Art der Arbeitsverhältnisse angeht, aber auch die Dominanz mittlerer fachlicher Qualifikationen. Dagegen werden einfachere Tätigkeiten oft ausgelagert bzw. durch Zeitarbeiter erledigt. Das Wachstum von zusätzlichen Arbeitsplätzen in den 2000er Jahren hat vor allem außerhalb des Normalarbeitsverhältnisses stattgefunden, jedoch nicht ausschließlich. Der Anstieg in atypischer Beschäftigung führt zu mehr Diversität auf dem deutschen Arbeitsmarkt bezüglich Entlohnung, Bestandsstabilität und der dominanten Arbeitszeitmodelle und ist Folge der Deregulierung am Rande des Arbeitsmarktes und des sektoralen Wandels. Es bestehen bemerkenswerte Unterschiede je nach Branche, Qualifikation und Berufsgruppen hinsichtlich Beschäftigungsstrukturen und -dynamiken, also auch unterschiedliche Formen und Grade der Entgrenzung. Vor allem bestimmte Dienstleistungen und Helfertätigkeiten sind Haupttätigkeitsfeld von atypisch Beschäftigten. Dieser Flexibilisierung des Arbeitsmarktes hat mehr Personen in bezahlte Tätigkeiten gebracht und die Beschäftigung von Geringqualifizierten hat sich zumindest stabilisiert. Zugleich sind die Unterschiede innerhalb des Arbeitsmarktes gewachsen. Die Marktkräfte - Angebot und Nachfrage nach bestimmten Qualifikationen wirken sich in einem flexibleren Gefüge stärker als früher auf die Art der Beschäftigung und die Entlohnung aus (Tabelle 4).

\section{Tabelle 4: Arbeitsbedingungen und Berufsgruppen}

\begin{tabular}{|c|c|c|}
\hline $\begin{array}{l}\text { Flexibilität } \\
\text { des (Teil) Arbeitsmarktes: } \\
\begin{array}{l}\text { Regulierung + gewerkschaftlichel } \\
\text { tarifliche Durchdringung }\end{array}\end{array}$ & Hoch & niedrig \\
\hline Hoch & eher „bad jobs“ & eher „good jobs“ \\
\hline Niedrig & $\begin{array}{l}\text { eher "good jobs“ (mit Neben- } \\
\text { wirkungen) }\end{array}$ & besonders viele "good jobs“ \\
\hline
\end{tabular}

Eigene Darstellung. 


\section{Triebkräfte und die Rolle von Reformen}

Zwar gilt noch immer das Normalarbeitsverhältnis als Maßstab, jedoch hat sich die Welt der Arbeit seit den 1970er oder 1980er Jahren massiv verändert. Es ist ein gewisser Trend zu einer mehr oder weniger stark polarisierten Struktur des Arbeitsmarktes zu beobachten, welcher von der Globalisierung, dem technologischen Wandel und von institutionellen Reformen getrieben wird. Während der technologische Fortschritt unter anderem viele Routinetätigkeiten automatisiert hat, führt der globale Wettberb zur Auslagerung mancher Teilschritte im Herstellungsprozess sowie insgesamt $\mathrm{zu}$ komplexen und internationalen Wertschöpfungsketten. Auch die Institutionen spielen eine bedeutende Rolle in der Entwicklung in Richtung unterschiedlicher Teilarbeitsmärkte. Seit Mitte der 1980er Jahre wurden flexible Erwebsformen stetig dereguliert. Besonders Anfang der 2000er als Zeitarbeit und Minijobs eingeführt wurden, sowie durch striktere Aktivierungspolitik vor allem in Folge der Hartz-Reformen. Weiterhin hat die tarifvertragliche Abdeckung abgenommen und die Flexibilität innerhalb der Tarifverträge zugenommen. Auch in Zukunft werden sich die Trends der Globalisierung und des technischer Fortschritts fortsetzen. Hinzu kommt der demographische Wandel, welcher Konsequenzen für die weitere Entwicklung von Arbeitsnachfrage, Arbeitsangebot und Arbeitsformen hat. Im Zuge des demographisch bedingten Fachkräftemangels werden die Unternehmen für begehrte Fachkräfte mehr Anstrengungen unternehmen, um attraktive Arbeitsbedingungen zu bieten und den Präferenzen der Beschäftigten zu entsprechen. Ein langfristiger Trend hin zu einer Ausdifferenzierung des Arbeitsmarktes ist erkennbar. Wissensintensive, kognitive und personenbezogende, soziale Tätigkeiten gewinnen an Bedeutung, während Routinetätigkeiten eher verlagert oder automatisiert werden. Flexible Arbeitsformen spielen in den Wachstumsberufen eine besondere Rolle, mit allen positiven und negativen Aspekten. 
3. Gestaltungsoptionen: Flexibilität und/oder Produktivität?

In der Regel reagiert der Staat auf hohe Arbeitslosigkeit mit Deregulierung auf dem Arbeitsmarkt, was in der weiteren Folge zu einer Expansion von atypischer Beschäftigung führt (Eichhorst und Marx 2011). Dagegen findet man ReRegulierungstendenzen bei niedriger Arbeitslosigkeit, wie etwa gegenwärtig. Ebenfalls werden Interventionen seitens des Staates wahrscheinlicher, wenn das allgemeine Unbehagen bezüglich der bestehenden Ungleichheit auf dem Arbeitsmarkt groß ist, aber bestimmte Fragen aufgrund der Schwäche der Sozialpartner nicht durch Tarifverträge gestaltet werden können, wie z.B. die Einführung des gesetzlichen Mindestlohnes zeigt.

Engere Grenzen des Arbeitsmarktes führen zu einer größeren Homogenität der Arbeitsbedingungen und stärken tendenziell die Anreize für produktivere Tätigkeiten und eine höhere Qualifikation der Beschäftigten. Eine Re-Regulierung atypischer und flexibler Erwerbsformen würde also auf der einen Seite den Zugang zum Arbeitsmarkt erschweren, jedoch die Qualität der verbleibenden Jobs vermutlich ein Stückweit verbessern. Wenn jedoch das Beschäftigungssystem wieder „exklusiver" wird, ist die Gefahr groß, dass Arbeitslose und Geringqualifizierte vermehrt vom Arbeitsmarkt ausgegrenzt werden und bestimmte Berufsbilder, die Einstiegsmöglichkeiten bieten, aber begrenzte Produktivitätspotenziale aufweisen, unter Druck geraten. Deswegen sollte atypische Beschäftigung eher so gestaltet werden, dass sie gleichzeitig als Einstieg in die Beschäftigung und als Sprungbrett dienen kann. Personen, die eine Erwerbstätigkeit aufnehmen, sollten durch atypische Beschäftigung eine Chance auf Einstieg und Einarbeitung erhalten, später dann aber auch unterstützende Qualifizierung und Übergangsmöglichkeiten erhalten, um ihre Chancen zu erhöhen in einem dauerhaften und besser bezahlten Arbeitsverhältnis zu arbeiten. Um dies zu erreichen, müssen die Rahmenbedingungen so verändert werden, dass sowohl Arbeitgeber als auch Arbeitnehmer Anreize zum Ein- und Aufstieg haben.

Anstatt flexible Arbeitsverträge generell $\mathrm{zu}$ begrenzen, ist eine sequentielle Heranführung von flexiblen Arbeitsverträgen an die Bedingungen im 
Normalarbeitsverhältnis zu bevorzugen, wohl wissend, dass in bestimmten Tätigkeiten flexible Arbeitsverträge von größerer Bedeutung sein müssen als in anderen. Hierbei ist das Modell der Minijobs in seiner aktuellen Gestalt durchaus in Frage zu stellen, da es gerade qualifizierte Frauen in oft nicht qualifikationsgerechten Jobs festhält. Die gegenwärtige Gesetzgebung sollte wenigstens so gestaltetet werden, dass geringfügige Beschäftigung nur befristet zugelassen wird und der Minijob lediglich für (Langzeit)Arbeitslose als Einstieg fungiert. Gleichzeitig sind die Hinzuverdienstgrenzen in der Grundsicherung zu verändern, und stets sollten Minijobs bei erwerbstätigen Hilfebeziehern mit einer Aktivierungsstrategie in Richtung substantiellerer Erwerbstätigkeit kombiniert werden. Außerdem sollte man für (Langzeit)Arbeitslose und erwerbstätige Geringqualifizierte realistische Aus- und Weiterbildungsprogramme umsetzen, um ihre Beschäftigungsfähigkeit zu erhöhen.

In Bezug auf die Übergänge zwischen befristeten und unbefristeten Verträgen sollten vor allem im öffentlichen und akademischen Bereich der Übergang erleichtert werden, indem der Bestandsschutz an die Regelungen des privaten Sektors angepasst wird, wo wir systematisch höhere Übernahmequoten beobachten.

Im Fall der Zeitarbeit steht eine Gesetzesänderung im Arbeitnehmerüberlassungsgesetz zur Diskussion, nämlich eine Einführung des „equal pay“ nach neun Monaten Einsatz im selben Entleihbetrieb und eine Begrenzung der Überlassungsdauer auf 18 Monate. Bei der Dauer von Zeitarbeitsverhältnissen in den Jahren 2000 bis 2012 lässt sich ein leichter Anstieg beobachten (Haller und Jahn 2014). So wuchs der Anteil an Zeitarbeitern, die länger als neun Monate beschäftigt waren, von knapp 22 Prozent im Jahr 2000 auf fast 28 Prozent in 2010 sowie der Anteil von Arbeitsverträgen mit einer Dauer von über 18 Monaten von rund 9 Prozent im Jahr 2000 auf beinahe 14 Prozent im Jahr 2010. Jedoch sind weiterhin die meisten Zeitarbeitsverhältnisse von kurzer Dauer. So waren im Jahr 2000 etwa 52 Prozent der Zeitarbeiter kürzer als drei Monate beschäftigt. Im Jahr 2011 galt dies immer noch für 47 Prozent. Damit wird deutlich, dass die aktuell geplanten Änderungen im Arbeitnehmerüberlassungsgesetz nur einen kleinen Teil der Zeitarbeiter betreffen. Dies kann zwar in einigen Fällen zu Verbesserungen führen, jedoch auch zu mehr Drehtüreffekten. Sinnvoller ist es, eine schrittweise „Equal pay“-Regelung einzuführen, etwa dem Modell tarifvertraglicher Regelungen folgend, als eine starre Frist hierbei und eine generelle 
Überlassungshöchstdauer. Bei der Re-Regulierung der Zeitarbeit stellt sich auch die Frage nach dem Verhältnis zu Werkverträgen, die in letzten Zeit auch teilweise kritisch bewertet worden sind, insofern sie möglicherweise zur Umgehung arbeitsund sozialrechtlicher Standards oder der Regeln in der Zeitarbeit genutzt werden. Werkverträge sind aber ein übliches Instrument der Kooperation zwischen Unternehmen bzw. internen und externen Partnern. Anstatt das Instrument der Werkverträge generell zu beschränken, sind klare und handhabbare Regeln sinnvoller, etwa bei der Definition von Selbstständigkeit.

Generell stellt sich die Frage, ob diese Anreize für beide Parteien (Arbeitnehmer und Arbeitgeber), atypische Beschäftigung als Einstieg in die Beschäftigung sowie als Sprungbrett zu gestalten, erfolgreicher durch gesetzliche Regelungen oder durch betriebliche bzw. sektorale Vereinbarungen erreicht werden können. Der Vorteil von gesetzlichen Regelungen ist, dass sie flächendeckend für alle gelten und in der Regel übersichtlicher sind. Auf der anderen Seite können sektorale oder betriebliche Vereinbarungen den Besonderheiten einzelner Wirtschaftszweige eher gerecht werden. Hierbei ist aber die Reichweite begrenzt, nämlich auf Bereiche mit starken Gewerkschaften, Betriebsräten und Arbeitgeberverbänden. Es bietet sich also an, bei allgemeinen gesetzlichen Regelungen Öffnungsklauseln für Branchen einzuräumen, die zu abweichenden tarifvertraglichen Regelungen kommen können. 


\section{Ausblick}

Durch die zunehmende Flexibilisierung des deutschen Arbeitsmarktes knnte die Beschäftigung im Dienstleistungssektor sowie die Integrationsfähigkeit des Arbeitsmarktes erhöht werden. Flexible Erwerbsformen (und interne Flexibilität) dürften auch künftige Arbeitsmarktentwicklung positiv beeinflussen, jedoch verschärft dies gleichzeitig die Unterschiedlichket der Arbeitsverhältnisse. Dies ist angesichts des Strukturwandels nicht völlig zu vermeiden, aber gleichwohl stellen sich Fragen einer angemessenen institutionellen Gestaltung der Erwerbsformen. Generell muss es künftig darum gehen, die Aufstiegschancen in bessere und stabilere Beschäftigung im Rahmen des Möglichen zu verbessern. Eine allgemeine ReRegulierung des Arbeitsmarktes ist durchaus kritisch $\mathrm{zu}$ bewerten, da sie Beschäftigungspotenziale vermindern und den Arbeitsmarkt wieder exklusiver machen könnte. Es wäre besser die Übergangschancen von Arbeitnehmern von atypischer Beschäftigung, verstanden als Einstiegsgelegenheiten, in anderen Erwerbsformen zu erhöhen. Dies setzt einige angemessene Veränderungen bei den Regeln atypischer Beschäftigung sowie eine intensivere berufliche Qualifikation voraus. 
Quellen:

Badura, B., Ducki, A., Schröder, H., Klose, J. und Meyer, M. (2012). FehlzeitenReport 2012: Gesundheit in der flexiblen Arbeitswelt: Chancen nutzen Risiken minimieren. Berlin, Heidelberg: Springer.

Bundesregierung (2013). Psychische Belastungen aufgrund flexibler und atypischer Arbeitszeiten. Antwort der Bundesregierung auf eine Kleine Anfrage. Deutscher Bundestag. Drucksache 17/12273.

DFK (2013). DFK-Erreichbarkeitsstudie. www.barmer-gek.de/503929.

DGB (2012). Arbeitshetze, Arbeitsintensivierung, Entgrenzung. Ergebnisse der Repräsentativumfrage 2011. http://www.dgb-index-gutearbeit.de/downloads/publikationen/data/arbeitshetze_arbeitsintensivierung_ent grenzung_-_ergebnisse_der_repraesentativumfrage_2011.pdf.

Dustmann, C., Ludsteck, J. und Schönberg, U. (2009). Revisiting the German Wage Structure. Quarterly Journal of Economics 124 (2): 843-888.

Eichhorst, W. und Tobsch, V. (2013). Has atypical work become typical in Germany? ILO Employment Working Paper 145. Genf.

Eichhorst, W. und Tobsch, V. (2014). Flexible Arbeitswelten. IZA Research Report 59.

Eichhorst, W. et al. (2012). Geringfügige Beschäftigung - Situation und Gestaltungsoptionen. IZA Research Report 47.

Eichhorst, W. und Marx, P. (2011). Reforming German Labour Market Institutions. A Dual Path to Flexibility. Journal of European Social Policy 21 (1): 73-87.

Gottschall, K. und Voß, G. G. (Hrsg.) (2005). Entgrenzung von Arbeit und Leben. Zum Wandel der Beziehung von Erwerbstätigkeit und Privatsphäre im Altag. 2. Aufl. München, Mering: R. Hampp Verlag.

Haller, P, und Jahn, E. (2014). Zeitarbeit in Deutschland: Hohe Dynamik und kurze Beschäftigungsdauern. IAB-Kurzbericht, 13/2014, Nürnberg, $10 \mathrm{~S}$.

Hammermann, A. und Stettes, O. (2013). Qualität der Arbeit - zum Einfluss der Arbeitsplatzmerkmale auf die Arbeitszufriedenheit im europäischen Vergleich. Köln: IW Trends 2/2013.

Hofmann, J., Rollwagen, I. und Schneider, S. (2007). Deutschland im Jahr 2020. DB Research.

Hofmann, J. und Nøstdal, R. (2014). Einsatz und Bedeutung externer Spezialisten. Fraunhofer IAO.

Klebe, T. (2013). Werkverträge: Verbreitung, Erscheinungsformen und rechtspolitischer Handlungsbedarf. Vortrag beim Symposium Werkverträge am 11.3.2013 in Berlin. 
Lohmann-Haislah, A. (2012). Stressreport Deutschland 2012 - Psychische Anforderungen, Ressourcen. Dortmund, Berlin, Dresden: Bundesanstalt für Arbeitsschutz und Arbeitsmedizin.

OECD (2013). OECD Employment Outlook. Paris.

Rump, J. et al. (2010). Betriebliche Projektwirtschaft. Eine Vermessung. Eine empirische Studie des Instituts für Beschäftigung und Employability im Auftrag von Hays.

Voß, G. G. (1998). Die Entgrenzung von Arbeit und Arbeitskraft. Eine subjektorientierte Interpretation des Wandels der Arbeit. Mitteilungen aus der Arbeitsmarkt- und Berufsforschung, 31 (3): 473-487. 